\title{
Suboptimal asthma control: prevalence, detection and consequences in general
} practice

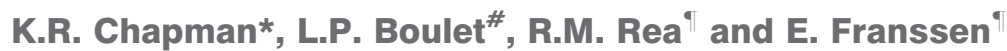

ABSTRACT: Telephone surveys describing suboptimal asthma control may be biased by low response rates.

In order to obtain an unbiased assessment of asthma control and assess its impact in primary care, primary care physicians used a 1-page control questionnaire in $\mathbf{5 0}$ consecutive asthma patients.

Of the 10,428 patients assessed by 354 physicians, $59 \%$ were uncontrolled, $19 \%$ well-controlled and $23 \%$ totally controlled. Physicians overestimated control, regarding only $42 \%$ of patients as uncontrolled. Physicians were more likely to report plans to alter the regimens of uncontrolled patients than controlled patients ( 1.29 versus 0.20 medication changes per patient) doing so in a fashion consistent with guideline recommendations. Of the uncontrolled patients, $59 \%$ required one or more urgent care or specialist visits versus 26 and $15 \%$ of well-controlled or totally controlled patients, respectively. Patients were more likely to report short-term symptom control when they had not required urgent or specialist care (odds ratio 5.68; $95 \%$ confidence interval 4.91-6.58).

The majority of asthma patients treated in general practice are uncontrolled. Lack of control can be recognised by physicians who are likely to consider appropriate changes to therapy. A lack of short-term symptom control of asthma is associated with excess healthcare utilisation.

KEYWORDS: Guideline dissemination, healthcare utilisation

$\mathbf{T}$ he assessment of asthma control has become pivotal in the management of asthma. However, several surveys in developed nations have shown that the majority of patients with asthma do not enjoy adequate asthma control [1-5]. This is not because asthma control is unrealistic and not achievable; the multicentre, multinational study of asthma management, the Gaining Optimal Asthma control (GOAL) [6] study, has shown that well-controlled asthma is achievable in the majority of patients. A plausible explanation for these findings is that the prevalence of poor asthma control has been overestimated by surveys that rely upon random digit telephone dialling techniques. In the Asthma in Canada survey [1], for example, 49,767 Canadian households were contacted to identify 801 adults with asthma and 200 parents of children with asthma willing to participate in the survey, a response rate of $2 \%$ as compared with a prevalence of asthma of $5-10 \%$ in the general population. Participation in the study was voluntary and it is possible that the study was skewed toward patients who had poor disease control.

It has also been suggested that there is a discrepancy between what is achievable and what is achieved in asthma management because patients appear to have low expectations for their own disease control. Among poorly controlled Canadian patients surveyed, $84 \%$ regarded their disease as adequately controlled or very well controlled [1]. Moreover, the physicians surveyed believe that the majority of their patients enjoy optimal control of their disease. The failure of patients to voice their concerns or to report troublesome symptoms and a low index of suspicion for poor control by physicians could account for the perpetuation of the current lack of asthma control [7]. Moreover, relatively little is known about how physicians evaluate control in

\section{AFFLLIATIONS}

*University of Toronto, Toronto,

\#Laval University, Quebec City, Quebec, and

"GlaxoSmithKline Canada Inc. Mississauga, Ontario, Canada.

\section{CORRESPONDENCE}

K.R. Chapman

Asthma and Airway Centre University Health Network Toronto Western Hospital Room 7E-451

399 Bathurst Street

Toronto

Ontario

M5T $2 S 8$

Canada

Fax: 14166033456

E-mail: kchapman@ca.inter.net

Received:

August 032007

Accepted after revision:

October 112007

STATEMENT OF INTEREST

None declared. 
their own practices. For these reasons, the present study was undertaken.

The present general practice study attempted to address the gap between asthma control achieved and asthma control achievable by developing a practice audit for physicians. Participating physicians surveyed consecutive patients with asthma, asking asthma symptom control questions and deciding each patient's control status and the steps most appropriate in subsequent management. Using this intervention: 1) an unbiased assessment of asthma control in the general practice setting was obtained; 2) the ability of primary care physicians to assess control in their own practices was determined; 3) the factors associated with sub-optimal control were determined; 4 ) the actions taken by physicians when they identify suboptimal control were determined; and 5) the healthcare utilisation impact of suboptimal symptom control was gauged.

\section{METHODS}

Primary care physicians from a national database were recruited to participate in a Personal Practice Assessment Programme as part of a nationally accredited continuing medical education programme. Physicians from urban and rural practices and from both anglophone and francophone communities were eligible to participate if they were in active non-specialty practice. Before patient data collection, physicians completed a written questionnaire concerning their management of asthma. They also received 50 one page questionnaires to allow them to record symptom control in the next consecutive 50 patients with asthma attending the practice (See supplementary data). Patients were eligible to participate if they had physician-diagnosed asthma and were $\geqslant 12$ yrs of age. Patients were eligible whether or not they were visiting the practice on that day for asthma care or for other reasons. Control symptom questions were based upon the Canadian consensus guidelines for the management of asthma and are outlined in table 1 [8]. Questions concerning the use of shortacting $\beta_{2}$-agonists and the frequency of daytime and night-time symptoms were asked in reference to the previous week. Questions concerning the limitation of activities and absence from work or school were made in reference to the previous 3 months. The patient questionnaire was modified after approximately half of the patient data had been collected to add three questions concerning healthcare utilisation for urgent asthma care in the previous year. Patients also responded to questions concerning their medication, action plans and education received for asthma self-care. After collection of the symptom control information, physicians were asked to indicate on the same questionnaire page whether or not they considered the patient's asthma to be under control and what further management steps they would consider, including medication prescriptions and follow-up. Physicians had been provided a copy of the Canadian consensus guidelines at the beginning of the study and were familiar with the general objectives and hypotheses of the study. Participating physicians gave written consent to participate in the programme and the programme was reviewed and approved by a national ethics review board.

Patients were classified as having uncontrolled asthma if they fell short of the acceptable levels of control on any of the five key symptom parameters outlined in table 1: daytime symptoms, sleep disturbances, physical activity limitations, absenteeism and use of relief medication. Asthma was considered controlled if patients did not exceed guideline recommendations for these parameters. Patients who did not provide information to evaluate control were excluded. For some analyses, patients were further categorised to denote those with no asthma symptoms as enjoying total control.

\section{Data analysis}

Relationships between physician characteristics and asthma control were explored by dividing the physicians into those who had more than the median percentage of uncontrolled patients and those who had the median percentage or less and establishing comparisons. Logistic regression analysis with backward selection was used to determine predictors of control. Variables included: sex; age; smoking status; selfreported compliance; inhaler instruction given; and whether or not lung function had been measured by the physician. Healthcare utilisation was analysed separately in a univariate model, as these data were collected only during the second half of the study. Odds ratios ORs) and confidence intervals (CIs) were calculated using logistic regression. A Chi-squared test was used to compare proportions. The statistical level of significance was 0.05 .

\section{RESULTS}

\section{Participating physicians}

In total, $81 \%$ of 570 physicians approached to participate did so. No difference could be detected between participating and nonparticipating physicians in terms of age, sex, type of practice

\section{TABLE 1 Criteria for asthma control}

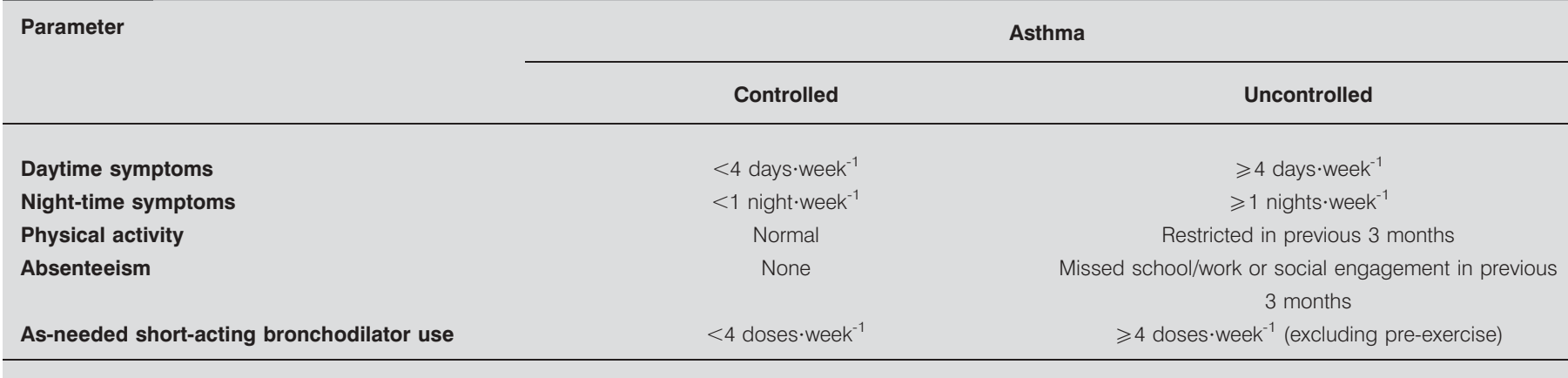




\begin{tabular}{|c|c|c|c|c|c|c|c|c|c|c|c|c|c|}
\hline & Subjects $n$ & 0 & $1-3$ & $4-7$ & $>7$ & 0 & $1-2$ & 3-4 & $5-7$ & 0 & $1-2$ & $3-4$ & $5-7$ \\
\hline Controlled & 4282 & 66 & 34 & 0 & 0 & 69 & 31 & 0 & 0 & 100 & 0 & 0 & 0 \\
\hline
\end{tabular}

or community of practice. Of the 462 physicians enrolled, 354 provided patient data, $22 \%$ were female, $25 \%$ were in solo practices, $28 \%$ practiced in towns of $<50,000$ inhabitants and $36 \%$ had graduated from medical school $>20$ yrs before study commencement. Median enrolment was 26 patients per physician. No differences in physicians' characteristics could be detected between practices with higher than median $(57 \%)$ or lower than median percentage of patients controlled.

\section{Patient characteristics}

Of the 10,428 patients surveyed, $60 \%$ were female. In total, $36 \%$ were $\leqslant 35$ yrs and $15 \%$ were $>65$ yrs of age. Patient socioeconomic data were not collected but all were receiving care through Canada's universal healthcare system and a selection of small community and urban practices increased the likelihood that the patients represented the spectrum of Canadian demographics. Of the patients approached to participate; $<3 \%$ declined. The majority of patients were

\begin{tabular}{|c|c|c|}
\hline \multirow[t]{2}{*}{ TABLE 3} & \multicolumn{2}{|c|}{$\begin{array}{l}\text { Patient characteristics positively and negatively } \\
\text { associated with achieving asthma control }\end{array}$} \\
\hline & & OR $(95 \% \mathrm{Cl})$ \\
\hline \multicolumn{3}{|c|}{ Positively associated } \\
\hline Male sex & & $1.15(1.05-1.25)$ \\
\hline \multicolumn{3}{|l|}{ Age yrs } \\
\hline $12-35$ ve & us $>65$ & $1.41(1.20-1.66)$ \\
\hline $19-35$ ve & us $>65$ & $1.34(1.17-1.54)$ \\
\hline $36-50$ ve & us $>65$ & $1.14(0.99-1.30)^{\#}$ \\
\hline $51-65$ ve & us $>65$ & $1.05(0.92-1.21)^{\#}$ \\
\hline \multicolumn{3}{|c|}{ Smoking status } \\
\hline Nonsmo & r versus current smoker & $2.60(2.34-2.90)$ \\
\hline Former $\mathrm{V}$ & sus current smoker & $2.23(1.95-2.54)$ \\
\hline \multicolumn{3}{|c|}{ Written action plan } \\
\hline Yes vers & no & $1.28(1.15-1.43)$ \\
\hline \multicolumn{3}{|c|}{ Following dosing instructions } \\
\hline "Always & ersus "When I need to" & $1.14(1.04-1.25)$ \\
\hline "Always & ersus "When I remember" & $1.92(1.64-2.23)$ \\
\hline \multicolumn{3}{|c|}{ Negatively associated } \\
\hline \multicolumn{3}{|c|}{ Demonstrated inhaler technique } \\
\hline Yes vers & & $0.90(0.82-0.98)$ \\
\hline \multicolumn{3}{|c|}{ Spirometry performed } \\
\hline Yes vers & no & $0.83(0.76-0.91)$ \\
\hline
\end{tabular}

OR: odds ratio; $\mathrm{Cl}$ : confidence interval; \#: nonsignificant. lifetime nonsmokers (56\%), while $24 \%$ were current smokers and $19 \%$ were former smokers. The majority of patients $(59 \%)$ were uncontrolled and $41 \%$ were controlled. Overall, 19\% were considered adequately controlled and $22 \%$ were considered totally controlled. The frequency of symptoms and bronchodilator use is shown in table 2. Limitation of physical activity due to asthma symptoms was reported by $45 \%$ of uncontrolled patients, and an absence from work, school or usual activities by $27 \%$ of uncontrolled patients.

Among all patients, 57\% were considered by their physicians to have nonasthma reasons for visiting the physicians' office, $26 \%$ were attending for routine asthma care and $16 \%$ were attending for urgent asthma care. Uncontrolled patients were more often considered to be making a visit for urgent asthma care needs compared with controlled patients (26 versus $2 \%$; $p<0.001)$. Similar proportions of controlled and uncontrolled patients were attending for routine asthma care. Approximately $20 \%$ of patients used inhaled short-acting $\beta_{2^{-}}$ agonist bronchodilators alone, 39\% used inhaled corticosteroid maintenance monotherapy, 35\% used inhaled corticosteroid and inhaled long-acting $\beta_{2}$-agonist maintenance therapy, $1 \%$ used maintenance leukotriene receptor antagonists, $2 \%$ used miscellaneous regimens and 3\% reported using no asthma medication at the time of the survey. There was no difference between controlled and uncontrolled patients with respect to the medications used for asthma management.

As shown in table 3, controlled asthma was positively associated with male sex, age $<35$ yrs, nonsmoking or exsmoking status, the availability of a written action plan and self-reported compliance with therapy. Conversely, controlled asthma was negatively associated with receiving inhaler instruction and with having had an objective measurement of lung function performed. Control was unrelated to physician characteristics including sex, size of practice and years since graduation from medical school.

Of the uncontrolled patients, 59\% required one or more urgent care or specialist visits, whereas only $26 \%$ of adequately controlled and $15 \%$ of totally controlled patients had required such care. Urgent office visits accounted for $63.1 \%$, emergency room visits for $20.2 \%$, specialist visits for $12.5 \%$ and hospitalisations for $4.2 \%$. Patients were more likely to report short-term symptom control of asthma when they had not required urgent or specialist care as compared with requiring one or more such visits (OR 5.68; 95\% CI 4.91-6.58). Comparing the types of urgent care needs between uncontrolled and controlled patients, unscheduled office visits for urgent care were reported by 47 

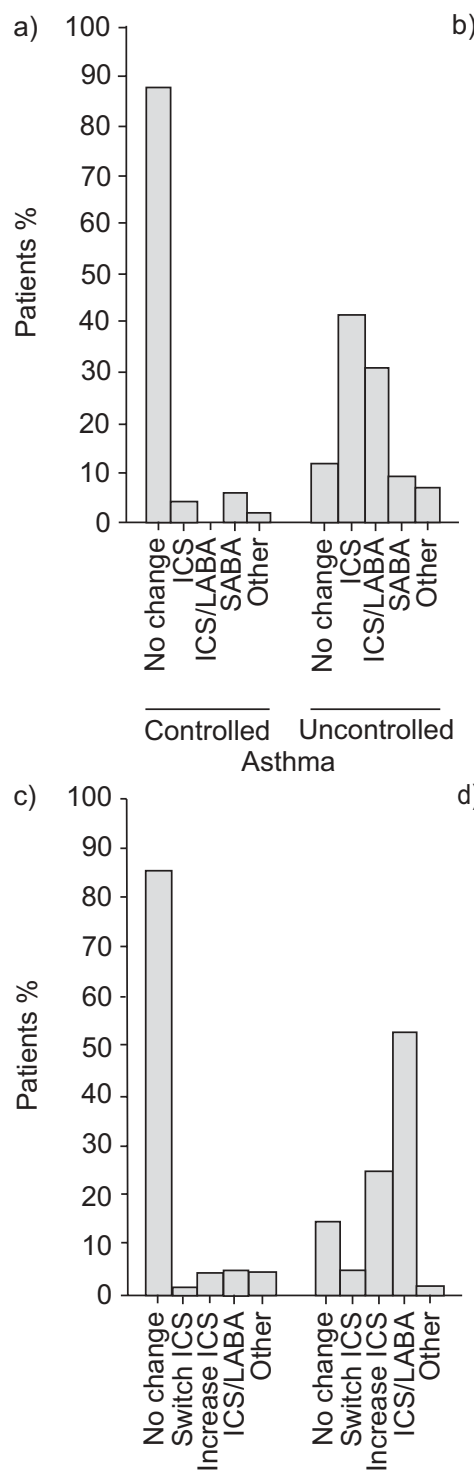

$\overline{\text { Controlled Uncontrolled }}$ Asthma
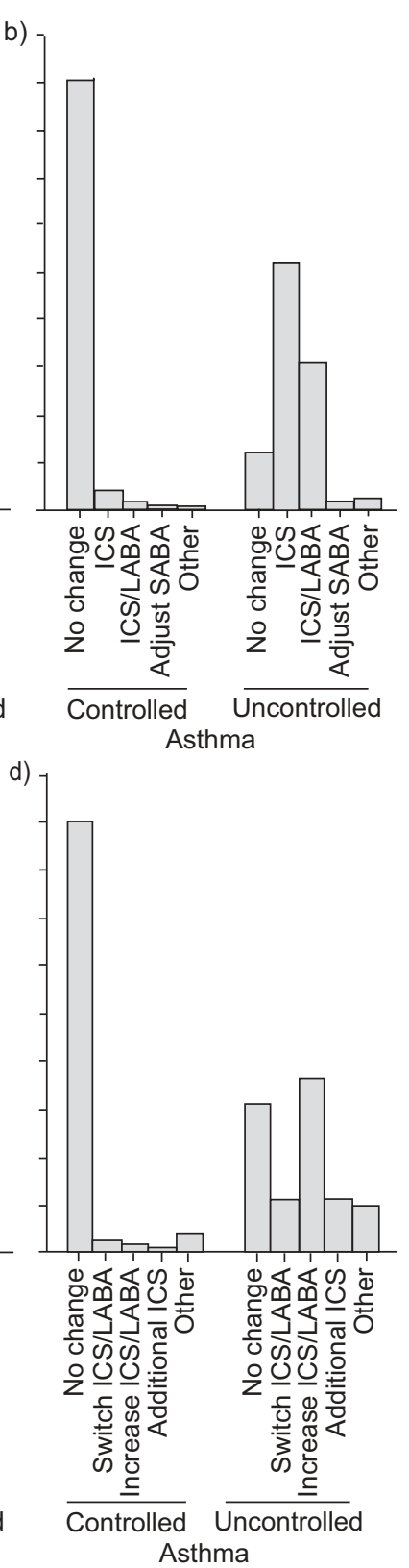

FIGURE 1. Medication changes recommended for controlled and uncontrolled patients using a) no medication, b) short-acting bronchodilators only, c) inhaled corticosteroid maintainence therapy without adjunctive maintainence medications and d) inhaled cortico and long-acting brochodilator maintainence therapy. ICS inhaled corticosteroids; ICS/LABA: combination therapy of ICS and long-acting $\beta$ agonists (LABA); SABA: short-acting $\beta$-agonists.

versus $13 \%$, emergency room visits by 15 versus $4 \%$ and hospital admission by 3 versus $0.6 \%$, for all $\mathrm{p}<0.001$.

When patients described their use of asthma medications, 47\% reported that they always did so, $10 \%$ did so "when they remembered" and $41 \%$ did so "only when [they] need to". Management shortcomings were commonly reported by patients; $80 \%$ did not have a written action plan to guide their handling of an exacerbation, 32\% had never demonstrated their inhaler use to a healthcare professional and $44 \%$ had never had spirometry.

\section{Physician assessment and proposed management of patients surveyed}

Physicians significantly overestimated control among their patients, regarding only $42 \%$ as uncontrolled. Physicians were discordant with guideline classification of control in $31 \%$ of uncontrolled patients, $13 \%$ of well-controlled patients and $2 \%$ of totally controlled patients. Most commonly, physicians were discordant with guideline criteria when patients showed lack of control in terms of only one parameter, most often the overuse of quick-relief medication.

Despite the under-recognition of uncontrolled asthma, physicians were more likely to manage uncontrolled patients differently from controlled patients. A follow-up visit within 3 months was requested for $69 \%$ of uncontrolled patients but for just $25 \%$ of controlled patients $(p<0.001)$. Physicians were more likely to report plans to alter the regimens of uncontrolled patients than controlled patients (1.29 versus 0.20 medication changes per patient, $\mathrm{p}<0.01)$. Among uncontrolled patients not using asthma medication at the time of the survey, the most frequently recommended change was the initiation of inhaled corticosteroids (41\% of such patients; fig. 1a). Similarly, among uncontrolled patients using only a quick reliever, initiating inhaled corticosteroid monotherapy as maintenance treatment was the most frequently recommended step (52\% of such patients; fig. $1 \mathrm{~b})$. Among patients already using an inhaled corticosteroid monotherapy, initiation of combination therapy was the most frequently recommended step (53\% of such patients; fig. 1c). For patients already taking combination therapy and classified as uncontrolled, an increase in the formulation strength or dosing schedule was the single most common recommendation (for 36\% of such patients; fig. 1d). Other changes, such as the prescription of prednisone, antileukotriene agents or theophylline, were infrequent (fig. 1a-d). For $18 \%$ of uncontrolled patients, physicians reported plans to schedule quick-acting bronchodilator use, confusing their role as quick relievers with that of maintenance medications.

\section{DISCUSSION}

The present findings confirm and extend the previous examinations of asthma control in practice settings. First, using a relatively unbiased general practice sample of patients in Canada, it has been confirmed that the majority of patients do not enjoy adequate guideline-defined asthma control. Secondly, it has been found that sub-optimal control is more likely in females, current smokers, older patients and those who lack a written action plan for exacerbation management. Thirdly, the lack of short-term symptom control is not a trivial or nonsignificant finding; patients lacking symptom control are far more likely to require urgent asthma care in the form of an emergency visit or hospitalisation than their counterparts who enjoy adequate guideline-defined asthma control. Finally, primary care physicians recognise a lack of control among most, but not all, of their uncontrolled asthma patients. Despite this imperfect assessment of control, primary care physicians are still likely to recommend appropriate medication changes and aftercare to patients who fail to achieve guideline targets for asthma control.

The methodology used herein overcomes the potential concern that telephone survey methodologies have overestimated the prevalence of poor control, a concern that is plausible if poorly 
controlled patients are more troubled by their asthma and more likely to take the time to respond to a telephone survey. Moreover, the survey used in the present study, undertaken by physicians themselves, offers greater confidence that the patients who are surveyed suffer from physician-diagnosed asthma and not from other respiratory diseases such as chronic obstructive pulmonary disease (COPD). It might be argued that surveying patients in a medical care setting has biased the study towards finding patients who lack adequate asthma control since such patients are more likely to require care and, in particular, urgent care. This is unlikely, however, because the proportion of patients who were uncontrolled was similar in all patients surveyed (59\%), all patients who had attended for routine assessment or nonasthma related visit (51\%) and only patients with asthma who had attended for nonasthma reasons (47\%).

The finding that females were less likely to have symptomcontrolled asthma than males is consistent with the observation that in asthma, as in several chronic disease settings, females may report symptoms differently from males, being more likely to volunteer symptoms of disease and to seek medical care $[9,10]$. However, physiological explanations are also of potential importance. Nonspecific bronchial hyperresponsiveness is found more commonly among females than males, in both general population surveys and selected smoking populations [11, 12]. The association between smoking and lack of asthma control is plausibly explained by the direct airway irritant effect of tobacco smoke and the more recently described corticosteroid resistance among patients with asthma who smoke $[13,14]$. Although the relationship between age and asthma is complex, the weak association described herein between increasing age and decreasing asthma control is consistent with descriptions of adult onset asthma gradually worsening in severity over time [15]. It might be argued that the present finding, linking current smoking and increasing age with poor control of asthma, could also reflect contamination of the patient population by patients who have COPD. In the absence of specific pulmonary function testing, this confounding factor cannot be ruled out but it is unlikely to be a major factor for several reasons. First, few patients in the survey were using inhaled anticholinergic agents, a common practice that may help to distinguish physicians' customary management of COPD from customary management of asthma [16]. Secondly, the increased risk of uncontrolled asthma was present in current smokers but not former smokers, an unlikely finding if the tobacco-related problem was persistent airflow limitation. Finally, more than half of the surveyed patients had undergone spirometry and had the results of this been more typical of COPD than asthma, the patients would not have been regarded by their physicians as asthma patients eligible for the survey.

The present finding of an association between written action plan availability and achieving asthma control is generally consistent with the benefit of action plans, as described in randomised trials $[17,18]$. It must be noted, however, that the availability of a written action plan may not account directly for the benefit of short-term asthma symptom control, but may be an indication that such patients have received more general asthma education or specialised care. In this context, it seems counterintuitive that demonstrating patients' inhaler technique to a healthcare professional is associated with a lower likelihood of achieving control, but the relationship is clearly not causal. This finding almost certainly indicates that teaching inhaler techniques is not a routine part of asthma care but is reserved for patients whose disease is more symptomatically troublesome. The present authors believe that similar reasoning accounts for the relationship between having had spirometry and having a higher likelihood of uncontrolled asthma [16, 19, 20].

The present authors believe that the current findings highlight one of the important factors responsible for the high prevalence of poorly controlled asthma; physicians' assessments of asthma control were not concordant with guideline assessment recommendations. Physicians often failed to identify as poorly controlled those patients who exceeded guideline parameters for adequate control, particularly the too frequent use of quick relief bronchodilators. The present authors suspect that this overestimate of asthma control occurs more commonly in actual practice settings than was revealed here because in this study, physicians were asked to focus on asthma control in their patients, were given questionnaires to guide them through control assessments and had previously been given a copy of the current Canadian asthma guidelines publication. The present study suggests that a systematic audit by primary care physicians of their own patients could lead to management changes consistent with guideline recommendations. If validated, this strategy could be used to improve the management of not only asthma but other chronic diseases.

Some limitations to the present study must be noted. First, the physicians who took part in the study were volunteering to participate in a continuing medical education programme and may represent a physician group less in need of educational intervention in asthma. Secondly, there is no way of knowing whether or not physicians acted upon the changes in management for uncontrolled patients that they had suggested during the study. Further research will be required to determine if guided assessment of asthma control in general practice can lead not only to changes in management but to improved outcomes. Finally, the present study used a definition of control based upon Canadian guidelines, a definition similar, but not identical, to the Global Iniative for Asthma guidelines. The simplified questionnaire used herein does not allow the recalculation of the prevalence of control by alternate definitions.

In conclusion, the current authors believe that the present study has confirmed the feasibility of studying asthma management practices in the primary care setting and, in doing so, has confirmed the high prevalence of uncontrolled asthma in Canada. Moreover, the process of guided assessment of asthma control leads to the identification of inadequate control by physicians and corresponding proposals to change medication therapy appropriately. Further study is warranted to determine if the practice audit can be used to improve the recognition and treatment of poorly controlled asthma, thereby helping to close the gap between guideline recommendations and guideline implementation.

\section{REFERENCES}

1 Chapman KR, Ernst P, Grenville A, Dewland P, Zimmerman S. Control of asthma in Canada: failure to 
achieve guideline targets. Can Respir J 2001; 8: Suppl. A, 35A-40A.

2 Adachi M, Morikawa A, Ishihara K. [Asthma insights \& reality in Japan (AIRJ).]. Arerugi 2002; 51: 411-420.

3 Adams RJ, Fuhlbrigge A, Guilbert T, Lozano P, Martinez F. Inadequate use of asthma medication in the United States: results of the asthma in America national population survey. J Allergy Clin Immunol 2002; 110: 58-64.

4 Lai CKW, De Guia TS, Kim YY, et al. Asthma control in the Asia-Pacific region: The Asthma Insights and Reality in Asia-Pacific Study. J Allergy Clin Immunol 2003; 111: 263-268.

5 Rabe KF, Vermeire PA, Soriano JB, Maier WC. Clinical management of asthma in 1999: the Asthma Insights and Reality in Europe (AIRE) study. Eur Respir J 2000; 16: 802-807.

6 Bateman ED, Boushey HA, Bousquet J, et al. Can guidelinedefined asthma control be achieved? The Gaining Optimal Asthma ControL study. Am J Respir Crit Care Med 2004; 170: 836-844.

7 Boulet LP, Phillips R, O'Byrne P, Becker A. Evaluation of asthma control by physicians and patients: comparison with current guidelines. Can Respir J 2002; 9: 417-423.

8 Boulet LP, Becker A, Bérubé D, Beveridge R, Ernst P. Canadian asthma consensus report, 1999: Canadian Asthma Consensus Group. CMAJ 1999; 161: Suppl. 11, S1-S61.

9 Osborne ML, Vollmer WM, Linton KL, Buist AS. Characteristics of patients with asthma within a large HMO: a comparison by age and gender. Am J Respir Crit Care Med 1998; 157: 123-128.

10 Tovt-Korshynska MI, Dew MA, Chopey IV, Spivak MY, Lemko IS. Gender differences in psychological distress in adults with asthma. J Psychosom Res 2001; 51: 629-637.
11 Manfreda J, Sears MR, Becklake MR, et al. Geographic and gender variability in the prevalence of bronchial responsiveness in Canada. Chest 2004; 125: 1657-1664.

12 Kanner RE, Connett JE, Altose MD, et al. Gender difference in airway hyperresponsiveness in smokers with mild COPD. The Lung Health Study. Am J Respir Crit Care Med 1994; 150: 956-961.

13 Tomlinson JE, McMahon AD, Chaudhuri R, Thompson JM, Wood SF, Thomson NC. Efficacy of low and high dose inhaled corticosteroid in smokers versus non-smokers with mild asthma. Thorax 2005; 60: 282-287.

14 Chalmers GW, Macleod KJ, Little SA, Thomson LJ, McSharry CP, Thomson NC. Influence of cigarette smoking on inhaled corticosteroid treatment in mild asthma Thorax 2002; 57: 226-230.

15 De Marco R, Locatelli F, Cerveri I, et al. Incidence and remission of asthma: a retrospective study on the natural history of asthma in Italy. J Allergy Clin Immunol 2002; 110: 228-235.

16 Kesten S, Chapman KR. Physician perceptions and management of COPD. Chest 1993; 104: 254-258.

17 Beasley R, Cushley M, Holgate ST. A self management plan in the treatment of adult asthma. Thorax 1989; 44: 200-204.

18 Côté J, Bowie DM, Robichaud P, Parent JG, Battisti L, Boulet LP. Evaluation of two different educational interventions for adult patients consulting with an acute asthma exacerbation. Am J Respir Crit Care Med 2001; 163: 1415-1419.

19 Chapman KR, Tashkin DP, Pye DJ. Gender bias in the diagnosis of COPD. Chest 2001; 119: 1691-1695.

20 Chapman KR, Senathirajah N. The impact of pulmonary function testing on the diagnosis of asthma by primary care practitioners. Am J Respir Crit Care Med 1999; 155: A889. 